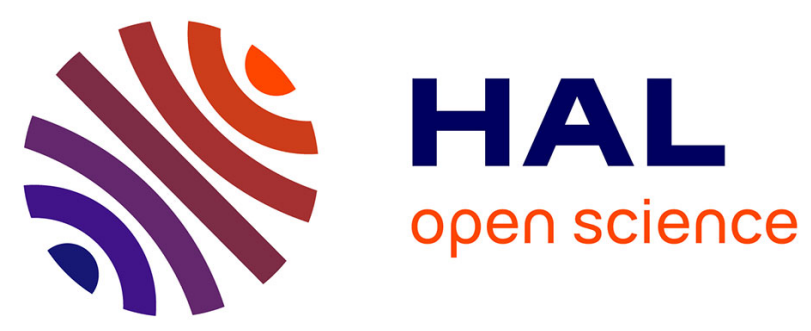

\title{
Effect of Mesh-to-Mesh Projection on the Magnetic Tooth Forces Calculation in Electrical Machines
}

Raphael Pile, Guillaume Parent, Yvonnick Le Menach, Jean Le Besnerais, Thomas Henneron, Jean-Philippe Lecointe

\section{- To cite this version:}

Raphael Pile, Guillaume Parent, Yvonnick Le Menach, Jean Le Besnerais, Thomas Henneron, et al.. Effect of Mesh-to-Mesh Projection on the Magnetic Tooth Forces Calculation in Electrical Machines. 2020 International Conference on Electrical Machines (ICEM), Aug 2020, Gothenburg (virtual), Sweden. pp.2500-2506, 10.1109/ICEM49940.2020.9271016 . hal-03203211

\section{HAL Id: hal-03203211 \\ https://hal-univ-artois.archives-ouvertes.fr/hal-03203211}

Submitted on 20 Apr 2021

HAL is a multi-disciplinary open access archive for the deposit and dissemination of scientific research documents, whether they are published or not. The documents may come from teaching and research institutions in France or abroad, or from public or private research centers.
L'archive ouverte pluridisciplinaire HAL, est destinée au dépôt et à la diffusion de documents scientifiques de niveau recherche, publiés ou non, émanant des établissements d'enseignement et de recherche français ou étrangers, des laboratoires publics ou privés. 


\title{
Effect of Mesh-to-Mesh Projection on the Magnetic Tooth Forces Calculation in Electrical Machines
}

\author{
Raphaël PILE, Guillaume PARENT, Yvonnick LE MENACH, Jean LE BESNERAIS, \\ Thomas HENNERON, and Jean-Philippe LECOINTE
}

\begin{abstract}
Magnetic forces calculation for the electromagnetic noise and vibration analysis in electrical machines (eNVH) is a key issue for an accurate modelling of magneto-mechanical interactions. An accurate method to compute magnetic forces consists in applying Virtual Work Principle (VWP). However, the magnetic force result depends intrinsically on the electromagnetic mesh which is generally not adapted to perform mechanical simulations. Thus, it may be necessary to perform mesh-to-mesh projection onto the mechanical mesh. In this paper, a 2D Ritz-Galerkin mesh-to-mesh projection is performed with a 12S10P Surface Permanent Magnet Machine (SPMSM). Only surface force density is considered. The novelty of the paper is to study the accuracy of this projection with respect to integrated tooth force. The results show that the mesh-to-mesh projection could be inaccurate in the tangential direction while accurate in the radial direction.
\end{abstract}

Index Terms-electrical machines, finite element, magnetic forces, mesh-to-mesh projection, vibrations.

\section{INTRODUCTION}

Electromagnetic Noise, Vibration \& Harshness (eNVH) in electrical machines is a growing issue related to automotive, health and comfort. This is due to the spreading use of electrical machines to replace internal combustion engines. As a consequence, the troubleshoot of eNVH issues at all design stages become an unavoidable step. In particular, the magneto-mechanical coupling is still an active research field.

A key point is the magnetic force computation. The airgap Maxwell tensor method is widely used to estimate the magnetic force based on the air-gap magnetic field knowledge for eNVH simulations [1]. However, the air-gap Maxwell tensor method does not represent a physical local force, but a physical global force when integrated along a closed contour in the air-gap. Moreover saturation can induce volume force density inside materials resulting in issues where applying force calculated with the Maxwell tensor [2]. Thus, for certain topologies, it will become necessary to use more precise methods to calculate magnetic forces.

R. Pile (email: raphael.pile@eomys.com) and J. Le Besnerais (email: jean.lebesnerais@eomys.com) are with EOMYS ENGINEERING company, Lille-Hellemmes 59260, France.

R. Pile, G. Parent (email: guillaume.parent@univ-artois.fr) and JeanPhilippe Lecointe (email: jphilippe.lecointe@univ-artois.fr) are with Univ. Artois, UR 4025, Laboratoire Systèmes Électrotechniques et Environnement (LSEE), F-62400 Béthune, France.

R. Pile, Y. Le Menach (email: yvonnick.le-menach@univ-lille.fr), and T. Henneron (email: thomas.henneron@univ-lille.fr) are with Univ. Lille, Arts et Metiers ParisTech, Centrale Lille, HEI, EA 2697 - L2EP - Laboratoire d'Electrotechnique et d'Electronique de Puissance, F-59000 Lille, France.

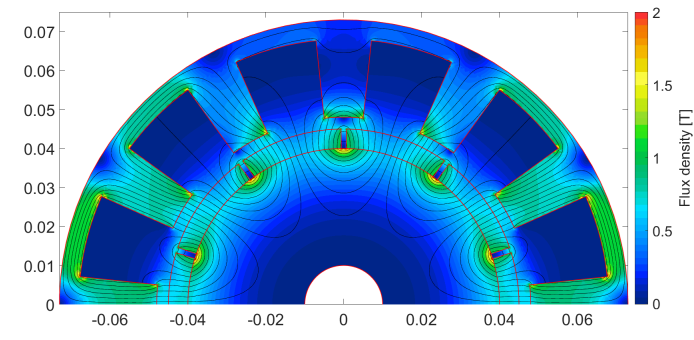

Fig. 1. Flux density and flux lines at no-load [3].

Instead, the Virtual Work Principle (VWP) can be used to compute accurately the local force on a Finite Element (FE) mesh [4]. The VWP calculations give a nodal resultant force which depends on the vicinity of the mesh [5]. This is an important restriction because the mesh used for electromagnetic model is not suitable for mechanical problem solving. Indeed, the electromagnetic mesh compared to the mechanical mesh is generally too thin on the tip of the teeth, and sometimes too coarse on the yoke. In this paper, a very thin magnetic mesh is used in order to accurately compute the magnetic forces.

The most accurate method to compute the equivalent magnetic forces on the mechanical mesh requires two numerical operations: first the magnetic force density must be computed, then this force density is projected on the mechanical mesh by a mesh-to-mesh projection approach (for example Ritz-Galerkin projection) [5]-[8]. However, previous works on the subject have validated this approach with a criterion adapted to the eNVH context.

Tooth forces are commonly used to study eNVH issues [9], because they ease mechanical model loading and physical interpretations through Fourier series development. In the event that distributed forces are to be used as in [8], this paper proposes to quantify the errors introduced by the meshto-mesh projection by comparing lumped tooth force values before and after projection. It defines an accuracy criterion for this type of projection which is adapted to the eNVH context.

The application is performed on a 12 slots and 10 poles (12S10P) Surface Permanent Magnet Synchronous Machine (SPMSM). An illustration of the magnetic flux density is presented in Fig. 1: flux density lines with high amplitude crossing ferromagnetic-air interface are linked to magnetic forces, such that a concentration of magnetic force on the tip of the teeth is expected. Thus, only magnetic surface force are considered in this work. The machine is an open-access 
testbench and all information can be obtained from [3]. This paper is a first step in order to propose a workflow based on the VWP to troubleshoot eNVH issues.

\section{Magnetic Forces Projection}

\section{A. Force Density Calculation}

According to [4], the resultant magnetic force $F_{s}^{i}$ (related to the spatial direction $s \in\{\mathbf{x}, \mathbf{y}, \mathbf{z}\}$ ) on a node can be calculated with a balance of magnetic co-energy on the elements $e$ surrounding this node:

$$
F_{s}^{i}=\sum_{\forall e \mid i \in e} \int_{e}\left(-\mathbf{B}^{T} \cdot \mathbb{J}^{-1} \cdot \frac{\partial \mathbb{J}}{\partial s} \cdot \mathbf{H}+\frac{\mu}{2}|\mathbf{H}|^{2}\left|\mathbb{J}^{-1}\right| \frac{\partial|\mathbb{J}|}{\partial s}\right) \mathrm{dV}
$$

where $\mathbf{H}$ is the magnetic field, $\mathbf{B}$ the magnetic flux density, and $\mathbb{J}$ the Jacobian matrix of each element. This integration is illustrated in Fig. 2. $F_{s}^{i}$ is expressed in Newton (or Newton per unit length in the case of a $2 \mathrm{D}$ calculation) and is proportional to the size of the surrounding elements: bigger elements implies more integrated magnetic co-energy.

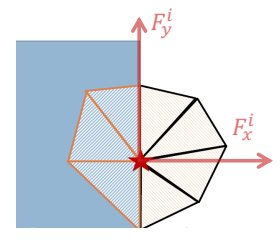

Fig. 2. Illustration of Virtual Work Principle integration on a node.

This is an issue when considering eNVH optimisation: a high number of mechanical simulation must be performed, and the mechanical numerical model must be accurately fitted. Since the magnetic mesh is often inappropriate for the mechanical simulation (too much elements and mesh density unsuitable to mechanical fitting), then it is common to perform a mesh-to-mesh projection using Ritz-Galerkin method for instance [5], [6]. Nevertheless, $F_{s}^{i}$ cannot be interpolated since it is the amplitude associated with a dirac function defined with respect to the point of application. The usual method to solve this issue is to compute an equivalent magnetic force density [5], [6]. The two step (computing density and applying mesh-to-mesh projection) are detailed in the Appendix V

To the authors knowledge, the error for resultant magnetic tooth force due to projection has not been studied in the literature. As a consequence, the Section III proposes to quantify these errors by comparing tooth forces before and after projection for the application case presented in Section II-B.

\section{B. Application with 12S10P SPMSM}

The studied topology is the same as in [3]. The magnetic field is solved using 2D non-linear magneto-static Finite Element Analysis (FEA) with MANATEE-FEMM coupling [10]. In this simulation, the magnetic saturation is very low such that only surface force can be considered. As expected,

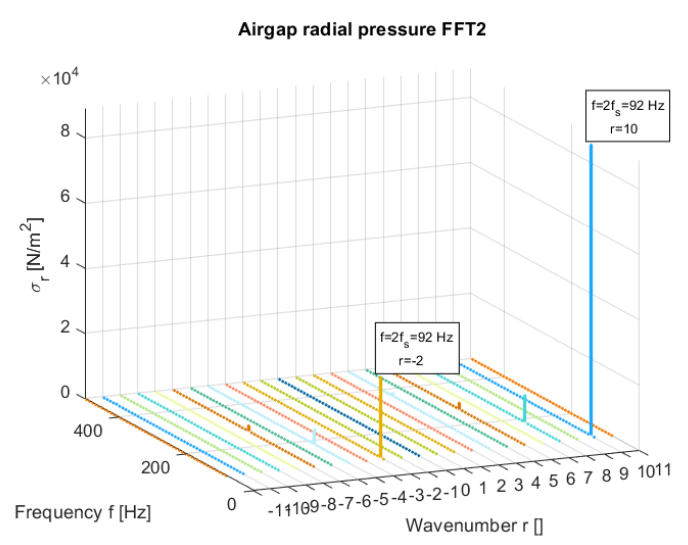

Fig. 3. Magnetic force spectrum computed with Maxwell Tensor at no-load.

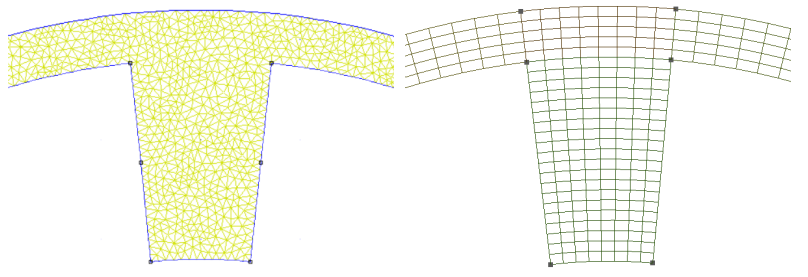

Fig. 4. Example of magnetic mesh (left) and a mechanical mesh with $\mathrm{N}_{\theta}=8$ angular divisions on the tooth tip (right).

the main magnetic force harmonic is at $2 f_{s}=92[\mathrm{~Hz}]$ as shown in Fig. 3. This frequency is used to test the proposed approach of mesh-to-mesh projection as it is known to be responsible for vibro-acoustic issues [3]. Moreover, the highest wave number $r=10$ at $2 f_{s}$ is the one most likely to introduce errors with sharp variations of the force density between nodes. The magnetic field at no-load is solved over 120 time steps during a full rotation of the rotor. A slidingband technique [11] is used such that the same mesh is kept at every time step. A comparison between the magnetic and mechanical mesh is presented in Fig. 4. The magnetic mesh has been highly refined to serve as a reference for forces calculation, but it is certainly not optimal (especially in the yoke). Unlike the magnetic mesh, the elements of the mechanical mesh are regularly spaced.

The magnetic forces are computed according to (1) for every time step. Then the time Fourier transform is computed for each nodal force. Thus, the magnetic nodal forces associated with frequency $2 f_{s}$ can be extracted as shown in Fig. 5.

The next step is to compute the magnetic surface force density. This requires to select all the "edges" at the airgapstator interface, and to built the corresponding basis of shape functions. At first, shape functions of order 1 are used (standard for 2D magnetic FEA), which are expressed in the reference element (see (14) in appendix). Then the matrix $[A]$ can be build and the linear system (5) can be solved. 


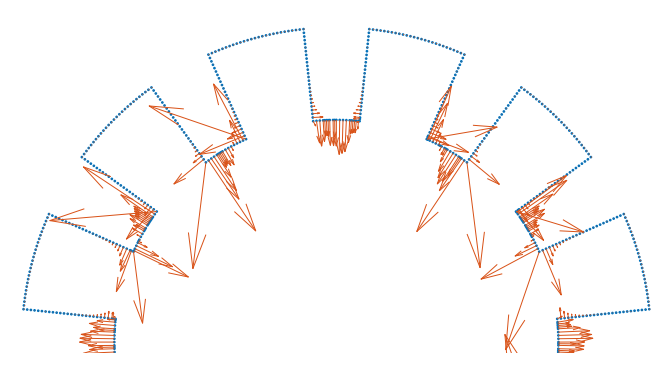

Fig. 5. Magnetic nodal forces $F_{s}$ calculated with the VWP at $2 f_{s}$.

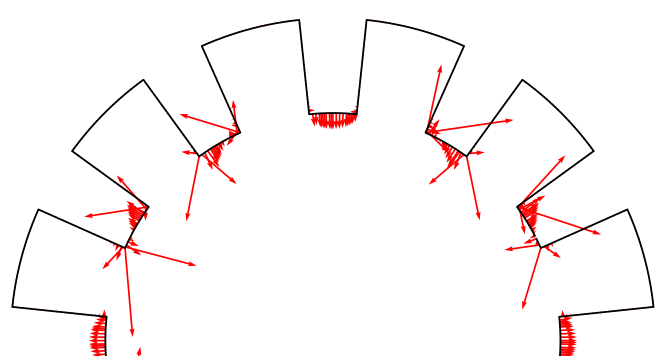

Fig. 6. Magnetic surface force density $f_{s}$ on the magnetic mesh at $2 f_{s}$.

The result is presented in Fig. 6: compared to Fig. 5, the distribution of force is smoothed as it is now displayed as a field. The dominant wavenumber $n=10$ predicted by the air-gap Maxwell Tensor theory in Fig. 3 can be observed by counting all the maxima in Fig.6.

Finally the mechanical mesh is read to get the new surface elements for the considered surface force. Thus, the shape functions in the reference element are the same as in (14). Then (11) and (13) can be calculated before solving (10). An example of the results computed with the mechanical mesh from Fig. 4 is presented in Fig. 7.

\section{Magnetic Tooth Force Projection}

\section{A. Objective}

The goal of this section is to study the accuracy of the mesh-to-mesh projection with respect to tooth forces. Tooth forces are defined as the sum of all nodal forces per tooth, and applied in the middle of the tooth tip as shown in Fig. 8. In all the following, the reference is the value of the tooth force computed on the magnetic mesh.

Indeed, tooth forces are commonly used to perform magneto-mechanical coupling [2], [9]. It is a powerful tool to have a first idea of the main eNVH sources. The mesh-tomesh projection may improve the accuracy of the magnetomechanical coupling. However, it is necessary to have at least a correct conservation of tooth forces and torque before any use of the results of mesh-to-mesh projection. This paper proposes to compare tooth forces before and after performing the mesh-to-mesh projection. An example of tooth force distribution is given in Fig. 8.

In order to simplify the parametrization of the mechanical mesh, $\mathrm{N}_{\theta}$ is defined as the number of divisions on the tip of the teeth. The other parameters of the mesh are calculated

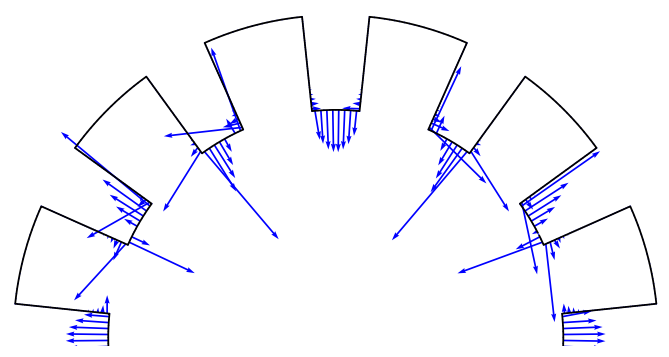

Fig. 7. Magnetic surface force density $\hat{f}_{s}$ on the mechanical mesh at $2 f_{s}$.

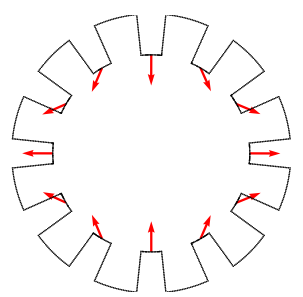

Fig. 8. Magnetic tooth forces on the mechanical mesh at $2 f_{s}$

from $\mathrm{N}_{\theta}$ such that:

$$
\begin{aligned}
& \mathrm{N}_{r, \text { yoke }}=\text { floor }\left\{\mathrm{N}_{\theta}\left(\mathrm{R}_{\mathrm{sy}}-\left(\mathrm{R}_{\mathrm{sbo}}+\mathrm{H}_{\mathrm{s}}\right)\right) / \mathrm{H}_{\mathrm{s}}+1\right\} \\
& \mathrm{N}_{r, \text { teeth }}=2 \mathrm{~N}_{\theta} \\
& \mathrm{N}_{\theta, \text { slot }}=\text { floor }\left\{1.2 \mathrm{~N}_{\theta}\right\}
\end{aligned}
$$

with $\mathbf{N}_{r, \text { yoke }}$ the number of divisions in the yoke thickness, $\mathrm{N}_{r, \text { teeth }}$ the number of divisions in the teeth height, and $\mathrm{N}_{\theta, \text { slot }}$ the number of angular divisions in the slots. The different possible results are illustrated in Fig. 9.

\section{B. Sensitivity to Gauss Points}

The first value to drive the precision is the number of Gauss points used in (13). This study is performed with the magnetic mesh in Fig. 4 and several mechanical mesh defined by the number of angular divisions $\mathrm{N}_{\theta}$. First the torque deviation after projection is illustrated in Fig. 10.

The direction of the magnetic forces is important to predict the resulting vibrations. In order to ease the physical interpretation, the magnetic forces are generally split into radial and tangential tooth forces defined from the radial direction in the middle of each tooth tip. The results are presented in Fig. 11 for the radial tooth force, and in Fig. 12 for the tangential tooth force. The projection has a more significant impact on the direction of the magnetic force: if the error on the radial force stay below $4 \%$ for all cases, the error on tangential force can reach up to $15 \%$ when the number of Gauss point is low. The error in the radial direction could correspond to $20 \log _{10}(1.04)=0.35$ [dB] in a vibroacoustic study, while the error in the tangential direction could correspond to $1.2 \mathrm{~dB}$. But with a high projection error, there is a risk of introducing new purely numerical spatial harmonics which can excite unexpected mechanical modes. This particular subject will be discuss in future research work.

The differences between radial and tangential errors can be explained by their spatial distribution: the tangential force 


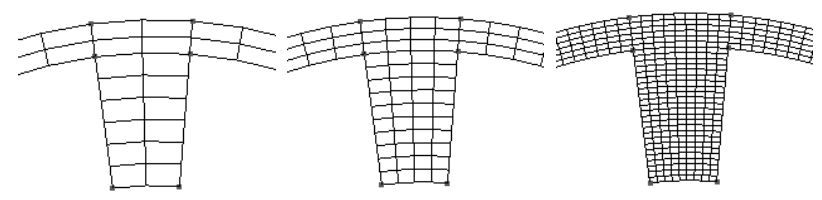

Fig. 9. Example of mechanical mesh with $\mathrm{N}_{\theta}=2, \mathrm{~N}_{\theta}=4$, and $\mathrm{N}_{\theta}=9$ angular divisions on the tooth tip.

Torque

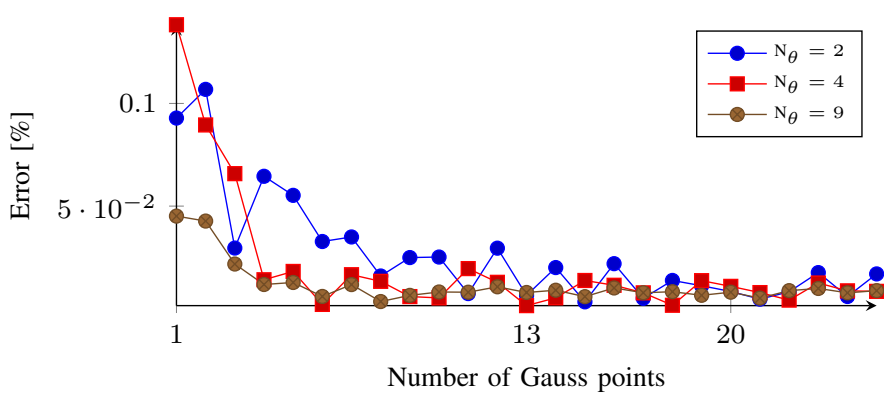

Fig. 10. Relative error for torque on the mechanical mesh compared to magnetic mesh.

are concentrated very close from the corners. Then the previous Gauss quadrature must be very accurate in this area to correctly capture the strong variations in the tangential force density. The radial force is more evenly distributed on tooth tip such that these error have less impact. In order to keep the error as small as possible, the choice of the number of Gauss points must be made according to the ratio of the mesh size. In this case, the thickest mechanical mesh with $\mathrm{N}_{\theta}=2$ angular divisions per tooth tip has approximately a size ratio of 1 for 6.5 elements compared to the magnetic mesh. In theory, two Gauss points are needed in each magnetic element to accurately compute the integrals [13]. But here 13 Gauss points does not ensure an exact integration because the Gauss points of the mechanical mesh do not generally match with the Gauss points of the magnetic mesh. This is illustrated by the small local increases in error along every plots in Fig. 1011-12.

Nevertheless, the error can be fairly reduced by increasing the number of Gauss points [7]. The computation cost of each Gauss point is pretty low because it does not influence the size of the system (10) to be solved. Thus a margin can be taken at relatively low cost.

\section{Sensitivity to Shape Function}

The shape functions used to calculate the force density on the mechanical mesh were chosen arbitrarily. It is therefore conceivable to artificially increase the order of these functions to see the effect on tooth force projection error. The mathematical definition of the order 2 used in the application is provided in the Appendix V. The comparison between the error from each order of shape function is presented in Fig. 13: there is no effect of the target shape function order on the accuracy for tooth force calculation, as it does not
Radial Tooth Force

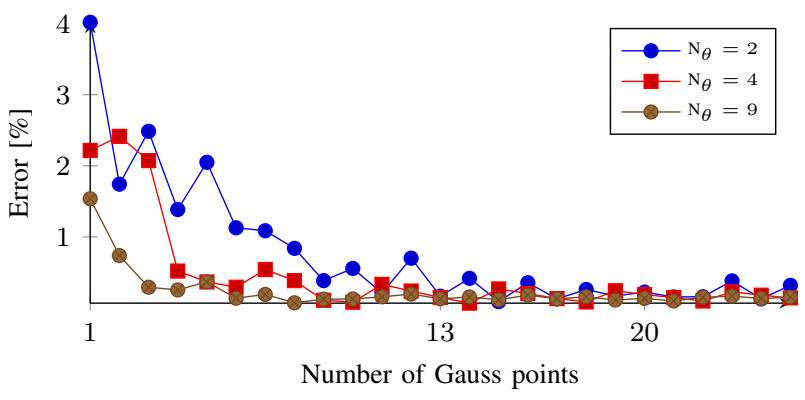

Fig. 11. Relative radial tooth forces error on the mechanical mesh compared to magnetic mesh.

Tangential Tooth Force

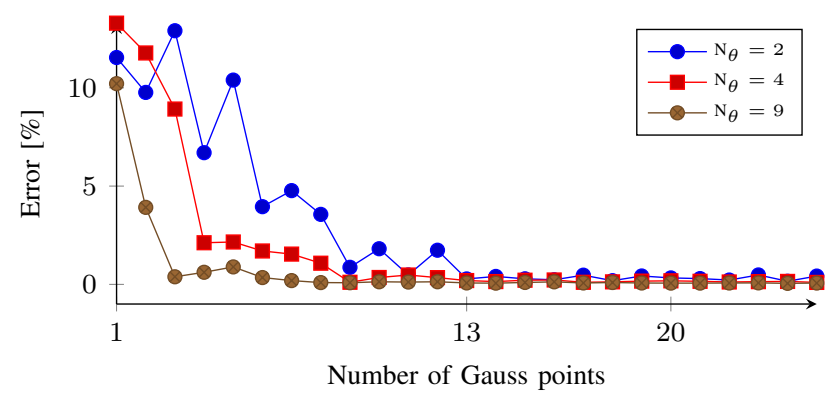

Fig. 12. Relative tangential tooth forces error on the mechanical mesh compared to magnetic mesh.

add any information to the solved system. The target shape function only have an impact on the calculation time. The errors can only be reduced with the interpolation of the force density (13).

Similarly, the shape function on the magnetic mesh are arbitrary chosen. However, it is not possible to increase the order of these shape function with classical order 2 such as (15) because the result of the VWP cannot be interpolated on intermediary nodes. The only remaining possibility is to increase the order of the used polynomials while keeping an element with two nodes. As example, one could build polynomial shape function defined on only 2 node per element. The mathematical definition of this pseudo-order 2 is provided in the Appendix V. Nevertheless, these polynomials lack appropriate mathematical properties to get a better accuracy on the field calculation, therefore also for the tooth forces calculation as observed in Fig. 14. One idea to explore would be to look for test functions in (7) ensuring the conservation of the nodal resultant forces from the magnetic mesh.

\section{Sensitivity to Mesh Size}

The third parameter to drive the precision is the mechanical mesh size. This study is performed by adjusting the mesh size as a function of angular division, while keeping the shape functions of order 1 for both meshes.

For the tooth forces, the results are presented in Fig. 16 for the radial direction, and in Fig. 17 for the tangential direction. In both cases, there is again a convergence of the 


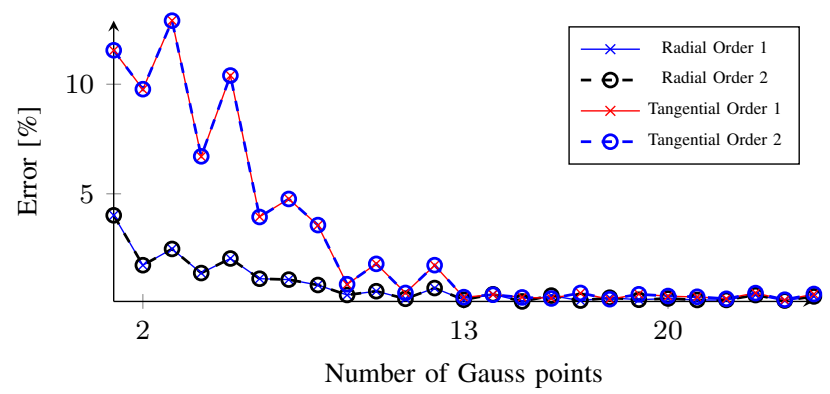

Fig. 13. Relative error for radial/tangential tooth forces on the mechanical mesh compared to magnetic mesh using different shape function orders on the target mechanical mesh $\left(\mathrm{N}_{\theta}=2\right)$.

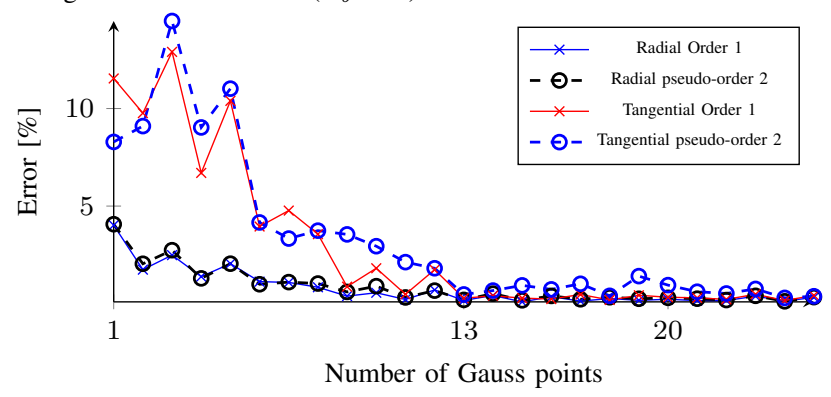

Fig. 14. Relative error for radial/tangential tooth forces on the mechanical mesh compared to magnetic mesh using different shape function orders on the source magnetic mesh $\left(\mathrm{N}_{\theta}=2\right)$.

error with the fineness of the mechanical mesh. However, this solution is much more expensive because not only it increases the size of the linear system (10) to be solved, but it also increases the computation time for solving the mechanical problem. It leads to the solving of two systems of complexity proportional to $N^{3}$ with $N$ the number of unknowns [14].

\section{CONCLUSION}

Noise and vibration from electromagnetic excitations in electrical machines rely on the accuracy of magnetic force calculation. Not only the magnetic force must be accurately computed on the magnetic model, but the results must be accurately projected onto the mechanical model.

In this paper the Ritz-Galerkin mesh-to-mesh projection technique was studied from a new angle: the accuracy for radial and tangential tooth forces was compared before and after projection. The sensitivity to the number of Gauss points, to nodal shape functions, and to mesh size were highlighted. It shows that most of the mesh-to-mesh projection errors concern tangential force: the parameters adequate for radial force projection might lack of accuracy regarding tangential force. One of the remaining issues to work on is the risk of exciting natural modes with numerical noise introduced/amplified by the projection.

This study is a first step before studying magnetomechanical coupling considering the relation between local force density and equivalent tooth forces. Future works will address mesh-to-mesh projection depending on the loading conditions (open-circuit, full-torque or field-weakening). Additionally, changing the loading conditions might introduce

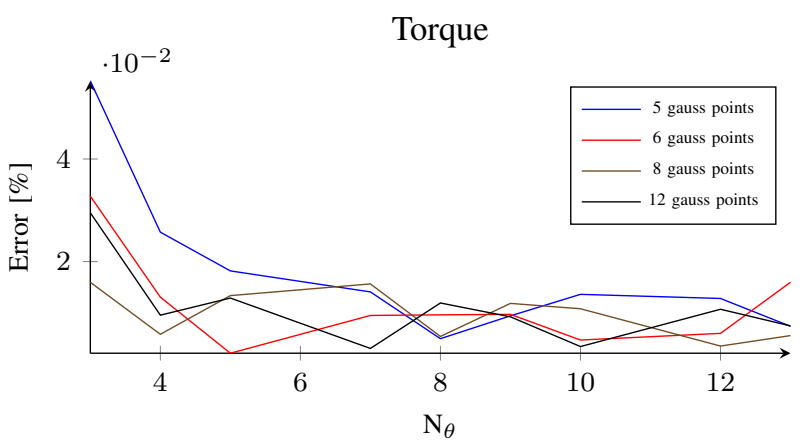

Fig. 15. Relative error depending on the mesh size for torque after meshto-mesh projection.

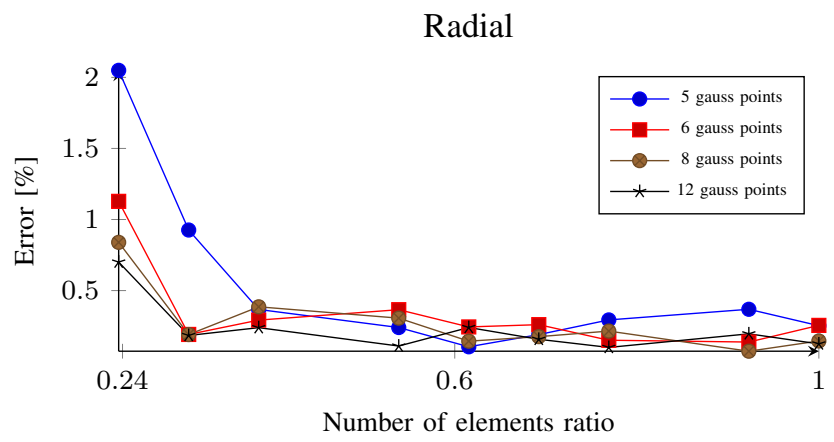

Fig. 16. Relative radial tooth forces error on the mechanical mesh compared to magnetic mesh depending on the relatives mesh size.

saturation effects, which raise the issue of magnetic volume force density effect on the vibro-acoustic results.

\section{APPENDIX}

\section{A. Calculating Force Density}

Considering that nodal resultants $F_{s}^{i}$ on a mesh comes from a continuous force field $f_{s}$ defined on a discrete shape function basis $\omega_{i}$ associated to the same mesh such that:

$$
f_{s}(x)=\sum_{k=1}^{N} \omega_{k}(x) f_{s, k}
$$

Then the link between $F_{s}^{i}$ and $f_{s}$ is the integration of the force field interpolated by all the shape functions [5]:

$$
\sum_{k=1}^{\mathrm{N}} f_{s, k} \int_{\mathrm{D}_{\mathrm{mag}}} \omega_{i} \omega_{k} d \tau=F_{s}^{i}
$$

where $\mathrm{D}_{\text {mag }}$ is the domain of definition in the magnetic source mesh, and $\mathrm{N}$ the total number of nodes. Considering all the possible combinations of $i$ and $k$, (4) leads to a linear matrix system of size $\mathrm{N}$ to be solved:

$$
[A]\left[f_{s}\right]=\left[F_{s}\right]
$$

with

$$
A_{i, k}=\int_{\mathrm{D}_{\operatorname{mag}}} \omega_{i} \omega_{k} d \tau
$$

Note that the choice of the basis $\omega_{i}$ is arbitrary. A natural choice for this basis $\omega_{i}$ is to use the electromagnetic FEA 


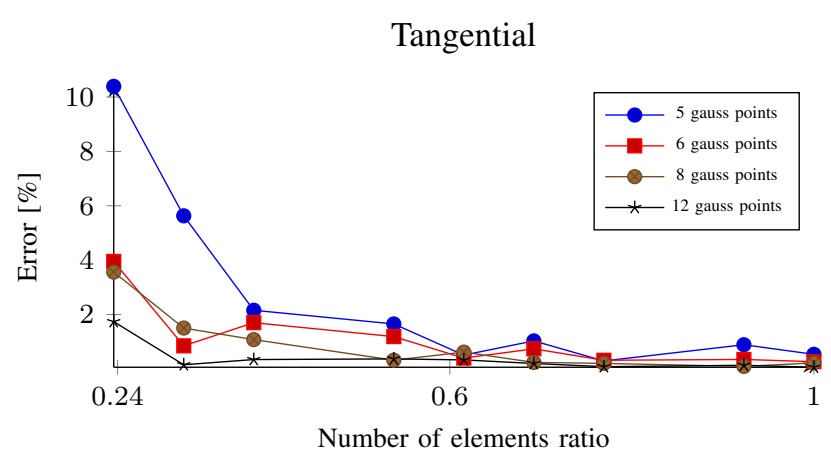

Fig. 17. Relative tangential tooth forces error on the mechanical mesh compared to magnetic mesh depending on the relative mesh size.

shape functions, which means for this study linear nodal shape functions.

At this point, the physical dimension $f_{s, k}$ depends on the types of elements which are considered. On one hand, $f_{s, k}$ is a surface force density when considering only surface elements in 3D (or edge elements in 2D). On the other hand, $f_{s, k}$ can be a volume force density considering volume elements in 3D (or facet elements in 2D). The choice depends on the loading condition of the electrical machines: with low magnetic saturation, magnetic forces concentrate at the ferromagnetic-air interface such that the surface force model is more accurate. However, a highly saturated case creates resultant forces from (1) inside the volume which can be then converted into volume force density.

\section{B. Mesh-to-Mesh Projection}

The goal of this section is to discuss the method to project the discrete magnetic force field $f_{s, k}$ on the mechanical mesh as a new discrete field $\hat{f}_{s, k}$. The criteria for the choice of methods concern precision, conservation of forces and calculation time.

Most of the mesh-to-mesh projection techniques rely on the Galerkin method because it ensures the uniqueness of the solution. Moreover the error is orthogonal to the chosen subspace [7], [15] and this ensures an accuracy that decreases with the size of the target mesh. The Galerkin method allows to obtain a new field distribution $\hat{f}_{s, k}$ on the mechanical mesh using a weak-formulation:

$$
\left\langle\psi, \hat{f}_{s, k}\right\rangle=\left\langle\psi, f_{s, k}\right\rangle
$$

where $\psi$ is a test function that belongs to a Sobolev space, and $\langle\cdot, \cdot\rangle$ a scalar product which corresponds to the same Sobolev space. With this weak-formulation, an error minimization of $\left\|\hat{f}_{s}-f_{s}\right\|$ is achieved in the sense of the norm defined on the Sobolev space. In this case, the choice of $\mathbf{L}^{2}$ for the Sobolev space is adapted for the conservation of the total force [6]:

$$
\left\|\hat{f}_{s}\right\|_{\mathbf{L}^{2}}=\int_{\mathrm{D}_{\text {mech }}} \hat{f}_{s}^{2} d \tau
$$

Indeed the integration of nodal forces (4) implicitly uses a

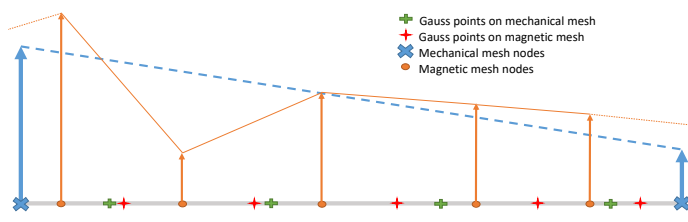

Fig. 18. Gauss quadrature between two 1D non-matching meshes.

$\mathbf{L}^{2}$ scalar product:

$$
\left\langle\hat{f}_{s, 1}, \hat{f}_{s, 2}\right\rangle_{\mathbf{L}^{2}}=\int_{\mathrm{D}_{\text {mech }}} \hat{f}_{s, 1} \hat{f}_{s, 2} d \tau
$$

Moreover, the input for the mechanical simulation is directly the projected force density (not its gradient/divergence/rotational). Therefore, the use of Hilbert spaces instead of $\mathbf{L}^{2}$ is useless in the context of force projection.

The test function $\psi$ must be chosen wisely in order to avoid ill-conditioned problem. Thus, the Ritz-Galerkin is often preferred [5]-[7]: the test function is also the target shape function basis of the mechanical mesh $\psi=\phi_{i}$.

Then, the previous weak-formulation can be written as another linear matrix system to be solved:

$$
[C][\hat{f}]=[f]
$$

with

$$
C_{i, k}=\int_{\mathrm{D}_{\text {mech }}} \phi_{i} \phi_{k} d \tau
$$

and

$$
f_{i}=\sum_{k=1}^{N} f_{s, k} \int_{\mathrm{D}_{\mathrm{mech}}} \phi_{i} \omega_{k} d \tau
$$

where $\mathrm{D}_{\text {mech }}$ is the domain of definition in the mechanical target mesh. The matrix $[C]$ is symmetric positive definite matrix so the problem (10) has a unique solution.

The calculation of each integral is performed using the Gaussian quadrature [13] on the mechanical mesh. Thus, the integration of (11) is accurate since Gaussian quadrature is exact for polynomials functions. However, the integration of (12) is not exact as illustrated in Fig. 18: seen by a mechanical mesh element, the force density field on the magnetic mesh is $\mathbf{C}^{1}$ piece-wise such that the Gaussian quadrature is only an approximation.

But, artificially increasing the number of Gauss points allows to reduce significantly the integration error with a low computational cost [7]. This solution is preferred because it is independent of the type of target and source meshes. Then, (12) can be approximated with a "high" $N_{g}$ number of Gauss points such that:

$$
f_{i} \approx \sum_{k=1}^{N} f_{s, k} \sum_{m=1}^{N_{g}} \phi_{i}\left(x_{m}\right) \omega_{k}\left(x_{m}\right) p_{m}
$$

with $x_{m}$ the Gauss points and $p_{m}$ Gauss weights. 


\section{Shape function}

Linear shape functions of the reference element of order 1 are defined as $\forall x \in[-1,1][12]$ :

$$
\left\{\begin{array}{l}
\omega_{1}(x)=(1-x) / 2 \\
\omega_{2}(x)=(1+x) / 2
\end{array}\right.
$$

Linear shape functions of the reference element of order 2 are defined as $\forall x \in[-1,1]$ [12]:

$$
\left\{\begin{array}{l}
\omega_{1}(x)=-(1 / 2)(1-x) x \\
\omega_{2}(x)=(1 / 2)(1+x) x \\
\omega_{3}(x)=(1+x)(1-x)
\end{array}\right.
$$

The polynomial "pseudo-order 2" shape functions are defined as $\forall x \in[-1,1]$ :

$$
\left\{\begin{array}{l}
\omega_{1}(x)=\left(1-x^{2}\right) / 2 \\
\omega_{2}(x)=\left(1+x^{2}\right) / 2
\end{array}\right.
$$

\section{REFERENCES}

[1] J. F. Gieras, C. Wang, and J. C. Lai, Noise of polyphase electric motors, CRC Press, Ed. CRC Press, 2006.

[2] R. Pile, E. devillers, and J. Le Besnerais, "Comparison of main magnetic force computation methods for noise and vibration assessment in electrical machines," IEEE Transactions on Magnetics, vol. 54, no. 7, pp. 1-13, July 2018.

[3] E. Devillers, M. Hecquet, X. Cimetiere, J. Lecointe, J. L. Besnerais, and T. Lubin, "Experimental benchmark for magnetic noise and vibrations analysis in electrical machines," in 2018 XIII International Conference on Electrical Machines (ICEM), Sep. 2018, pp. 745-751.

[4] J. Coulomb and G. Meunier, "Finite Element Implementation of Virtual Work Principle for Magnetic or Electric Force and Torque Computation," IEEE Transactions on Magnetics, vol. 20, no. 5, pp 1894-1896, sep 1984

[5] G. Parent, P. Dular, J.-P. P. Ducreux, and F. Piriou, "Using a galerkin projection method for coupled problems," IEEE Transactions on Magnetics, vol. 44, no. 6, pp. 830-833, jun 2008.

[6] A. A. Journeaux, F. Bouillault, and J.-Y. Roger, "Multi-physics problems computation using numerically adapted meshes: application to magneto-thermo-mechanical systems," Eur. Phys. J. Appl. Phys., vol. 61, no. 3, p. 30001, 2013.

[7] Z. Wang, T. Henneron, P. Dular, J.-C. Mipo, and F. Piriou, "Comparison of implementation techniques for galerkin projection between different meshes," International Journal of Numerical Modelling: Electronic Networks, Devices and Fields, vol. 27, no. 3, pp. 517-526, 2014.

[8] P. Kotter, D. Morisco, M. Boesing, O. Zirn, and K. Wegener, "Noisevibration-harshness-modeling and analysis of a permanent-magnetic disc rotor axial-flux electric motor," IEEE Transactions on Magnetics, vol. 54, no. 3, pp. 1-4, March 2018.

[9] A. Saito, M. Kuroishi, and H. Nakai, "Vibration Prediction Method of Electric Machines by using Experimental Transfer Function and Magnetostatic Finite Element Analysis," Journal of Physics: Conference Series, vol. 744, no. 1, 2016.

[10] EOMYS ENGINEERING, "MANATEE software: Magnetic acoustic noise analysis tool for electrical engineering (v1.09.01)," 2020 [Online]. Available: https://www.eomys.com/produits/manatee/article/ manatee-software

[11] D. Meeker, "FEMM (Finite Element Method Magnetics) v4.2," 2019 [Online]. Available: http://www.femm.info/

[12] J. Delmas, "Code aster: Functions of form and points of integration finite elements," 2017. [Online]. Available: https://code-aster.org/

[13] A. H. Stroud and D. Secrest, "Gaussian quadrature formulas," 1966

[14] A. Krishnamoorthy and D. Menon, "Matrix inversion using cholesky decomposition," in Signal Processing: Algorithms, Architectures, Arrangements, and Applications (SPA). IEEE, 2013, pp. 70-72.

[15] A. Bossavit, "How weak is the "weak solution" in finite element methods?" IEEE Transactions on Magnetics, vol. 34, no. 5, pp. 2429 2432, Sep. 1998.

\section{BIOGRAPHIES}

Raphaël Pile received a M.Sc. degree in Aerospace Engineering from ISAESupaéro, Toulouse, France and a M.Sc. degree in Fundamental and Applied Mathematics from Univ. Paul Sabatier, Toulouse, France, both in 2017. He is now working on an industrial $\mathrm{PhD}$ thesis at the L2EP laboratory (Univ. Lille, France) and LSEE laboratory (Univ. Artois, France) with the company EOMYS ENGINEERING. His PhD thesis focuses on the numerical methods to perform magneto-mechanical coupling for eNVH analysis.

Jean Le Besnerais currently works in EOMYS ENGINEERING as CEO and R\&D engineer. Following a M.Sc. specialized in Applied Mathematics (Ecole Centrale Paris, France) in 2005, he made an industrial $\mathrm{PhD}$ thesis in Electrical Engineering at the L2EP laboratory on the reduction of electromagnetic noise and vibrations in traction induction machines with ALSTOM Transport.

Guillaume Parent received the Ph.D. degree in Electrical Engineering jointly from the Univ. Lille and the Univ. Liège (Belgium), in 2008. He is currently a full Professor in electrical engineering at the LSEE laboratory. His main research interests include electromagnetic analysis with the finite element method applied to electromagnetic devices design.

Yvonnick Le Menach received his $\mathrm{PhD}$ in Electrical Engineering in 1999 (Univ. Lille). From 1999 to 2002 he was assistant professor in Univ. Artois. Since 2002, he is working as assistant professor in the L2EP laboratory. His research work concerns the numerical techniques for the Finite Integration Technique and the Finite Element Method applied to the electromagnetic systems.

Thomas Henneron received the Ph.D. degree in Electrical Engineering in 2004 (Univ. Lille). He is currently an Associate Professor at the L2EP. His research interests mainly concern the developement of model order reduction approaches applied to finite element models.

Jean-Philippe Lecointe received the $\mathrm{PhD}$ degree from the Univ. Artois (France) in 2003. He is currently Full Professor at the Artois University and he is the head of the LSEE laboratory (Univ. Artois, France). His research interests focus on electromagnetic design, efficiency, noise and vibrations of electrical machines. 